\title{
Symbolic Computation and Construction of New Exact Traveling Wave Solutions to Fitzhugh-Nagumo and Klein-Gordon Equations
}

\author{
Turgut Öziş ${ }^{\mathrm{a}}$ and İsmail Aslan ${ }^{\mathrm{b}}$ \\ ${ }^{a}$ Department of Mathematics, Ege University, 35100, Bornova, İzmir, Turkey \\ ${ }^{\mathrm{b}}$ Department of Mathematics, Izmir Institute of Technology, 35430, Urla, İzmir, Turkey \\ Reprint requests to T. Ö.; E-mail: turgut.ozis@ege.edu.tr
}

Z. Naturforsch. 64a, 15 - 20 (2009); received June 2, 2008 / revised July 16, 2008

\begin{abstract}
With the aid of the symbolic computation system Mathematica, many exact solutions for the Fitzhugh-Nagumo equation and the Klein-Gordon equation with a quadratic nonlinearity are constructed by an auxiliary equation method, the so-called $\left(G^{\prime} / G\right)$-expansion method, where the new and more general forms of solutions are also obtained. Periodic and solitary traveling wave solutions capable of moving in both directions are observed.
\end{abstract}

Key words: Auxiliary Equation Method; $\left(G^{\prime} / G\right)$-Expansion Method; Traveling Wave Solutions; Fitzhugh-Nagumo Equation; Klein-Gordon Equation.

\section{Introduction}

Explicit exact solutions of nonlinear partial differential equations may well describe different phenomena in physics and other fields. With the help of these solutions scientists are able to give better insight into the physical aspects of problems, and the construction of exact solutions of nonlinear partial differential equations plays an important role in solving nonlinear problems. During the past four decades searching for explicit solutions of nonlinear evolution equations by using a range of different methods was the main purpose for many researchers, and many powerful methods to construct exact solutions of nonlinear evolution equations have been established and developed, such as the homogeneous balance method [1], inverse scattering method [2], Hirota's bilinear method [3], Bäcklund transformation [4], F-expansion method [5], symmetry method [6], sine(cosine)function [7], $\tanh (\operatorname{coth})$-function [8], Painlevé expansion method [9], Homotopy Perturbation method [10], Exp-function method [11]. But there is no unified method that can be used to treat all types of nonlinear evolution equations, and the majority of the wellknown methods involve tedious computation if they are performed by hand.

In recent years, directly seeking for explicit exact solutions of nonlinear partial differential equations has attracted much attention, due to the availability of symbolic computation systems like Mathematica, Maple or Matlab which enable us to perform the complex and tedious computation by computer. Very recently, Wang et al. [12] introduced an auxiliary equation method, the so-called $\left(G^{\prime} / G\right)$-expansion method, which is easily carried out by computer, to look for traveling wave solutions of nonlinear evolution equations. Next, Bekir [13] obtained traveling wave solutions of some nonlinear evolution equations by using this method. Later, a generalization of this method has been introduced by Zhang et al. [14]. Moreover, Zhang et al. [15] gave a further extension of this method by applying it to the modified Korteweg-de Vries (mKdV) equation with variable coefficients.

The $\left(G^{\prime} / G\right)$-expansion method is based on the explicit linearization of nonlinear differential equations for traveling waves with a certain substitution which leads to a second-order differential equation with constant coefficients. The traveling wave solutions can be expressed by a polynomial in $\left(G^{\prime} / G\right)$, where $G=$ $G(\xi)$ satisfies a second-order linear ordinary differential equation [see (4)], $G^{\prime}=\frac{\mathrm{d} G(\xi)}{\mathrm{d} \xi}$, where $\xi=x-$ $\omega t$. The degree of the polynomial can be determined by considering the homogeneous balance between the highest-order derivatives and nonlinear terms appearing in a given nonlinear evolution equation, and the coefficients of the polynomial can be obtained by solving a set of algebraic equations resulted from the process of using the proposed method. The future of the $\left(G^{\prime} / G\right)$ - 
expansion method is briefly summarized in [12,13]. The computations are performed with the aid of a symbolic computation system like Mathematica, Maple or Matlab which enable us to perform the complex and tedious computation by computer.

In this paper, we will show, with the aid of the symbolic computation system Mathematica, the applicability of the $\left(G^{\prime} / G\right)$-expansion method to the FitzhughNagumo equation and the Klein-Gordon equation with a quadratic nonlinearity to construct many new and more general traveling wave solutions, soliton-like solutions and periodic solutions.

\section{Applications}

Periodic wavetrains are found in many systems. They are basic solutions corresponding to elementary solutions in the form of sinusoidal wavetrains, and the dispersion relation does not depend on the wave amplitude. In the nonlinear theory, the solutions are no longer sinusoidal, but periodic solutions may still exist. The main nonlinear effect of periodic solutions is not the difference in the functional form; it is the appearance of an amplitude dependence in the dispersion relation. This leads to a new qualitative behaviour, not merely to the correction of the dispersion relation in the linear case. Nonlinear dispersion problems are difficult to solve. Although an exact analytical solutions can be obtained, some nonlinear problems often appear in terms of very complicated implicit functions, which are not convenient for applications. Therefore, in this section, we would like to use the $\left(G^{\prime} / G\right)$-expansion method to obtain new and more general exact traveling wave solutions of two physically important nonlinear equations.

\subsection{The Fitzhugh-Nagumo Equation}

The Fitzhugh-Nagumo equation is a special case of the Burgers-Huxley equation

$$
u_{t}+\gamma u u_{x}-v u_{x x}=\beta u(1-u)(u-\alpha),
$$

where $\gamma, \beta \geq 0, \alpha(0<\alpha<1)$, and $v$ are parameters describing the interaction between convection, diffusion and reaction. When $\gamma=0$, the Burgers-Huxley equation reduces to the Hodgkin and Huxley equation which describes nerve pulse propagation in nerve fibers and wall motion in liquid crystals. Because of the complexity of the Huxley equation, Fitzhugh-Nagumo proposed simple, analytically tractable, and particularly useful model equations which contain the key features of the Huxley model. On the other hand, when $\beta=0$, the equation reduces to the Burgers equation describing diffusive waves in nonlinear dissipating systems [16].

Let us now consider the celebrated FitzhughNagumo equation in the form

$$
u_{t}-u_{x x}=u(1-u)(u-\alpha), \quad 0<\alpha<1,
$$

which occurs also in the theory of shallow water waves and plays an important role in the modeling of many physical phenomena such as plasma waves and magneto-acoustic waves.

Now, substituting $u(x, t)=u(\xi), \xi=x-\omega t$, in the diffusion equation (1) one obtains

$$
u^{\prime \prime}+\omega u^{\prime}-\alpha u+(1+\alpha) u^{2}-u^{3}=0,
$$

where the prime denotes the derivative with respect to $\xi$. For (2), we follow [9] and make an ansatz in the finite series form

$$
u(\xi)=\sum_{i=0}^{N} a_{i}\left(\frac{G^{\prime}(\xi)}{g(\xi)}\right)^{i},
$$

where $a_{i}$ are constants to be determined, $N$ is a positive integer which is determined by the homogeneous balancing method, and $G(\xi)$ is the solution of the auxiliary linear second-order ordinary differential equation

$$
G^{\prime \prime}(\xi)+\lambda G^{\prime}(\xi)+\mu G(\xi)=0,
$$

in which $\lambda$ and $\mu$ are also constants to be determined later. Thus balancing the highest-order derivative term $u^{\prime \prime}$ with the nonlinear term $u^{3}$ in (2) yields the leading order $N=1$. Therefore, we can write the solution of (2) in the form

$$
u(\xi)=a_{0}+a_{1}\left(\frac{G^{\prime}}{G}\right),
$$

where $G=G(\xi)$. Now, using (4) and (5) we derive that

$$
\begin{aligned}
u^{\prime}(\xi)= & -a_{1}\left(\frac{G^{\prime}}{G}\right)^{2}-\lambda a_{1}\left(\frac{G^{\prime}}{G}\right)-a_{1} \mu, \\
u^{\prime \prime}(\xi)= & 2 a_{1}\left(\frac{G^{\prime}}{G}\right)^{3}+3 a_{1} \lambda\left(\frac{G^{\prime}}{G}\right)^{2} \\
& +\left(a_{1} \lambda^{2}+2 a_{1} \mu\right)\left(\frac{G^{\prime}}{G}\right)+a_{1} \lambda \mu,
\end{aligned}
$$




$$
\begin{aligned}
u^{2}(\xi)= & a_{1}^{2}\left(\frac{G^{\prime}}{G}\right)^{2}+2 a_{1} a_{0}\left(\frac{G^{\prime}}{G}\right)+a_{0}^{2}, \\
u^{3}(\xi)= & a_{1}^{3}\left(\frac{G^{\prime}}{G}\right)^{3}+3 a_{1}^{2} a_{0}\left(\frac{G^{\prime}}{G}\right)^{2} \\
& +3 a_{1} a_{0}^{2}\left(\frac{G^{\prime}}{G}\right)+a_{0}^{3} .
\end{aligned}
$$

Substituting (5)-(9) into (2) and setting the coefficients of $\left(\frac{G^{\prime}}{G}\right)^{i}(i=0,1,2,3)$ to zero, we get the following set of over-determined algebraic equations for $a_{0}, a_{1}, \omega, \lambda$ and $\mu$ :

$$
\begin{aligned}
\left(\frac{G^{\prime}}{G}\right)^{0}: & a_{1} \lambda \mu-\omega \mu a_{1}-\alpha a_{0} \\
& +(1+\alpha) a_{0}^{2}-a_{0}^{3}=0 \\
\left(\frac{G^{\prime}}{G}\right)^{1}: & \left(2 a_{1} \mu+a_{1} \lambda^{2}\right)-\omega \lambda a_{1}-\alpha a_{1} \\
& +2(1+\alpha) a_{0} a_{1}-3 a_{0}^{2} a_{1}=0
\end{aligned}
$$

$$
\begin{gathered}
\left(\frac{G^{\prime}}{G}\right)^{2}: 3 a_{1} \lambda-\omega a_{1}+(1+\alpha) a_{1}^{2}-3 a_{1}^{2} a_{0}=0, \\
\left(\frac{G^{\prime}}{G}\right)^{3}: 2 a_{1}-a_{1}^{3}=0 .
\end{gathered}
$$

Solving the system (10) - (13) with the aid of Mathematica, we have the following three sets of solutions:

$$
\begin{gathered}
\omega=\mp \frac{\sqrt{2}(2 \alpha-1)}{2}, \quad \mu=\frac{a_{0}\left(a_{0}-1\right)}{2}, \\
\lambda=\mp \frac{\sqrt{2}\left(2 a_{0}-1\right)}{2}, \quad a_{1}=\mp \sqrt{2} ; \\
\omega=\mp \frac{\sqrt{2}(\alpha+1)}{2}, \quad \mu=\frac{\alpha-(1+\alpha) a_{0}+a_{0}^{2}}{2}, \\
\lambda=\mp \frac{1+\alpha-2 a_{0}}{\sqrt{2}}, \quad a_{1}= \pm \sqrt{2} ; \\
\omega=\mp \frac{\sqrt{2}(\alpha-2)}{2}, \quad \mu=\frac{a_{0}\left(a_{0}-\alpha\right)}{2}, \\
\lambda= \pm \frac{2 a_{0}-\alpha}{\sqrt{2}}, \quad a_{1}= \pm \sqrt{2} .
\end{gathered}
$$

Now, observing that $\lambda^{2}-4 \mu=\frac{1}{2}$ in the solution set (14), then solving (4) for $G(\xi)$ and plugging the obtained solution together with (14) into (5), we get the hyperbolic function traveling wave (soliton-like) solutions

$$
u_{1,2}(x, t)=\frac{1}{2}\left[1 \mp \frac{C_{1} \cosh \left(\frac{1}{2 \sqrt{2}}\left(x \pm \frac{\sqrt{2}(2 \alpha-1)}{2} t\right)\right)+C_{2} \sinh \left(\frac{1}{2 \sqrt{2}}\left(x \pm \frac{\sqrt{2}(2 \alpha-1)}{2} t\right)\right)}{C_{1} \sinh \left(\frac{1}{2 \sqrt{2}}\left(x \pm \frac{\sqrt{2}(2 \alpha-1)}{2} t\right)\right)+C_{2} \cosh \left(\frac{1}{2 \sqrt{2}}\left(x \pm \frac{\sqrt{2}(2 \alpha-1)}{2} t\right)\right)}\right],
$$

where $C_{1}$ and $C_{2}$ are arbitrary constants. By the same procedure, since $\lambda^{2}-4 \mu=\frac{1}{2}(\alpha-1)^{2}$ in the solution set (15), we derive the hyperbolic function traveling wave (soliton-like) solutions

$$
u_{3,4}(x, t)=\frac{\alpha+1}{2} \mp \frac{(\alpha-1)}{2}\left[\frac{C_{1} \cosh \left(\frac{1-\alpha}{2 \sqrt{2}}\left(x \pm \frac{\sqrt{2}(\alpha+1)}{2} t\right)\right)+C_{2} \sinh \left(\frac{1-\alpha}{2 \sqrt{2}}\left(x \pm \frac{\sqrt{2}(\alpha-1)}{2} t\right)\right)}{C_{1} \sinh \left(\frac{1-\alpha}{2 \sqrt{2}}\left(x \pm \frac{\sqrt{2}(\alpha+1)}{2} t\right)\right)+C_{2} \cosh \left(\frac{1-\alpha}{2 \sqrt{2}}\left(x \pm \frac{\sqrt{2}(\alpha+1)}{2} t\right)\right)}\right]
$$

where $C_{1}$ and $C_{2}$ are arbitrary constants. Lastly, since $\lambda^{2}-4 \mu=\frac{\alpha^{2}}{2}$ in the solution set (16), we end up with the following hyperbolic function traveling wave (soliton-like) solutions:

$$
u_{5,6}(x, t)=\frac{\alpha}{2}\left[1 \pm \frac{C_{1} \cosh \left(\frac{\alpha}{2 \sqrt{2}}\left(x \pm \frac{\sqrt{2}(\alpha-2)}{2} t\right)\right)+C_{2} \sinh \left(\frac{\alpha}{2 \sqrt{2}}\left(x \pm \frac{\sqrt{2}(\alpha-2)}{2} t\right)\right)}{C_{1} \sinh \left(\frac{\alpha}{2 \sqrt{2}}\left(x \pm \frac{\sqrt{2}(\alpha-2)}{2} t\right)\right)+C_{2} \cosh \left(\frac{\alpha}{2 \sqrt{2}}\left(x \pm \frac{\sqrt{2}(\alpha-2)}{2} t\right)\right)}\right],
$$

where $C_{1}$ and $C_{2}$ are arbitrary constants.

In particular, if we take $C_{2} \neq 0, C_{1}^{2}<C_{2}^{2}$, then (17) - (19) give the following solitary waves:

$$
u_{1,2}(x, t)=\frac{1}{2}\left[1 \mp \tanh \left(\frac{1}{2 \sqrt{2}}\left(x \pm \frac{\sqrt{2}(2 \alpha-1)}{2} t\right)+\xi_{0}\right)\right]
$$




$$
\begin{aligned}
& u_{3,4}(x, t)=\frac{\alpha+1}{2} \mp \frac{(\alpha-1)}{2} \tanh \left(\frac{1-\alpha}{2 \sqrt{2}}\left(x \pm \frac{\sqrt{2}(\alpha+1)}{2} t\right)+\xi_{0}\right), \\
& u_{5,6}(x, t)=\frac{\alpha}{2}\left[1 \pm \tanh \left(\frac{\alpha}{2 \sqrt{2}}\left(x \pm \frac{\sqrt{2}(\alpha-2)}{2} t\right)+\xi_{0}\right)\right]
\end{aligned}
$$

where $\xi_{0}=\tanh ^{-1}\left(C_{1} / C_{2}\right)$, representing the kink solitons.

\subsection{The Klein-Gordon Equation with a Quadratic Nonlinearity}

The Klein-Gordon equation is considered one of the most important mathematical models in quantum field theory. The equation appears in relativistic physics and is used to describe dispersive wave phenomena in general. In addition, it also appears in nonlinear optics and plasma physics. The Klein-Gordon equation arises in physics in linear and nonlinear forms. The nonlinear form comes from the quantum field theory and describes nonlinear wave interactions [17]. As an example, we consider the Klein-Gordon equation with quadratic nonlinearity in the form

$$
u_{t t}-\alpha^{2} u_{x x}+\beta u-\gamma u^{2}=0,
$$

where $\alpha, \beta$ and $\gamma$ are nonzero arbitrary constants.

The approach is similar to the scheme used in Section 2.1, so we skip the details. Letting $u(x, t)=u(\xi)$, $\xi=x-\omega t$ in (23), we get

$$
\left(\omega^{2}-\alpha^{2}\right) u^{\prime \prime}+\beta u-\gamma u^{2}=0 .
$$

Balancing the terms $u^{\prime \prime}$ and $u^{2}$ in (24) yields the leading-order $N=2$. Therefore, we can write the so- lution of (24) in the form

$$
u(\xi)=a_{0}+a_{1}\left(\frac{G^{\prime}}{G}\right)+a_{2}\left(\frac{G^{\prime}}{G}\right)^{2} .
$$

Substituting (25) into (24), setting the coefficients of $\left(\frac{G^{\prime}}{G}\right)^{i}(i=0,1, \ldots, 4)$ to zero, and solving the system of five algebraic equations for $a_{0}, a_{1}, a_{2}, \omega, \lambda$ and $\mu$ with the aid of Mathematica, we find the following two sets of solutions:

$$
\begin{aligned}
& a_{0}=\frac{3\left(\beta+\left(\omega^{2}-\alpha^{2}\right) \lambda^{2}\right)}{2 \gamma}, \quad a_{1}=\frac{6\left(\omega^{2}-\alpha^{2}\right) \lambda}{\gamma}, \\
& a_{2}=\frac{6\left(\omega^{2}-\alpha^{2}\right)}{\gamma}, \quad \mu=\frac{-\beta-\left(\omega^{2}-\alpha^{2}\right) \lambda^{2}}{4\left(\alpha^{2}-\omega^{2}\right)} ; \\
& a_{0}=\frac{-\beta+3 \lambda^{2}\left(\omega^{2}-\alpha^{2}\right)}{2 \gamma}, \quad a_{1}=\frac{6\left(\omega^{2}-\alpha^{2}\right) \lambda}{\gamma}, \\
& a_{2}=\frac{6\left(\omega^{2}-\alpha^{2}\right)}{\gamma}, \quad \mu=\frac{\beta+\left(\alpha^{2}-\omega^{2}\right) \lambda^{2}}{4\left(\alpha^{2}-\omega^{2}\right)} .
\end{aligned}
$$

Since $\lambda^{2}-4 \mu=\beta /\left(\alpha^{2}-\omega^{2}\right)$ in the solution set (26), we get the following solitary wave and periodic solutions:

$$
\begin{aligned}
& u_{1}(x, t)=\frac{3 \beta}{2 \gamma}\left[1-\left(\frac{C_{1} \cosh \left(\frac{1}{2} \sqrt{\frac{\beta}{\alpha^{2}-\omega^{2}}}(x-\omega t)\right)+C_{2} \sinh \left(\frac{1}{2} \sqrt{\frac{\beta}{\alpha^{2}-\omega^{2}}}(x-\omega t)\right)}{C_{1} \sinh \left(\frac{1}{2} \sqrt{\frac{\beta}{\alpha^{2}-\omega^{2}}}(x-\omega t)\right)+C_{2} \cosh \left(\frac{1}{2} \sqrt{\frac{\beta}{\alpha^{2}-\omega^{2}}}(x-\omega t)\right)}\right)^{2}\right], \\
& \beta\left(\alpha^{2}-\omega^{2}\right)>0,
\end{aligned}
$$$$
u_{2}(x, t)=\frac{3 \beta}{2 \gamma}\left[1+\left(\frac{-C_{1} \sin \left(\frac{1}{2} \sqrt{\frac{-\beta}{\alpha^{2}-\omega^{2}}}(x-\omega t)\right)+C_{2} \cos \left(\frac{1}{2} \sqrt{\frac{-\beta}{\alpha^{2}-\omega^{2}}}(x-\omega t)\right)}{C_{1} \cos \left(\frac{1}{2} \sqrt{\frac{-\beta}{\alpha^{2}-\omega^{2}}}(x-\omega t)\right)+C_{2} \sin \left(\frac{1}{2} \sqrt{\frac{-\beta}{\alpha^{2}-\omega^{2}}}(x-\omega t)\right)}\right)^{2}\right],
$$$$
\beta\left(\alpha^{2}-\omega^{2}\right)<0,
$$ 
where $C_{1}$ and $C_{2}$ are arbitrary constants. Similarly, since $\lambda^{2}-4 \mu=\beta /\left(\omega^{2}-\alpha^{2}\right)$ in the solution set (27), we derive the following solitary wave and periodic solutions:

$$
\begin{aligned}
& u_{3}(x, t)=\frac{\beta}{2 \gamma}\left[-1+3\left(\frac{C_{1} \cosh \left(\frac{1}{2} \sqrt{\frac{\beta}{\omega^{2}-\alpha^{2}}}(x-\omega t)\right)+C_{2} \sinh \left(\frac{1}{2} \sqrt{\frac{\beta}{\omega^{2}-\alpha^{2}}}(x-\omega t)\right)}{C_{1} \sinh \left(\frac{1}{2} \sqrt{\frac{\beta}{\omega^{2}-\alpha^{2}}}(x-\omega t)\right)+C_{2} \cosh \left(\frac{1}{2} \sqrt{\frac{\beta}{\omega^{2}-\alpha^{2}}}(x-\omega t)\right)}\right)^{2}\right], \\
& \beta\left(\omega^{2}-\alpha^{2}\right)>0, \\
& u_{4}(x, t)=\frac{\beta}{2 \gamma}\left[-1-3\left(\frac{-C_{1} \sin \left(\frac{1}{2} \sqrt{\frac{-\beta}{\omega^{2}-\alpha^{2}}}(x-\omega t)\right)+C_{2} \cos \left(\frac{1}{2} \sqrt{\frac{-\beta}{\omega^{2}-\alpha^{2}}}(x-\omega t)\right)}{C_{1} \cos \left(\frac{1}{2} \sqrt{\frac{-\beta}{\omega^{2}-\alpha^{2}}}(x-\omega t)\right)+C_{2} \sin \left(\frac{1}{2} \sqrt{\frac{-\beta}{\omega^{2}-\alpha^{2}}}(x-\omega t)\right)}\right)^{2}\right], \\
& \beta\left(\omega^{2}-\alpha^{2}\right)<0,
\end{aligned}
$$

where $C_{1}$ and $C_{2}$ are arbitrary constants.

In particular, if we take $C_{2} \neq 0 C_{1}^{2}<C_{2}^{2}$, then the solution functions (28) and (30) become

$$
\begin{aligned}
& u_{1}(x, t)=\frac{3 \beta}{2 \gamma} \operatorname{sech}^{2}\left(\frac{1}{2} \sqrt{\frac{\beta}{\alpha^{2}-\omega^{2}}}(x-\omega t)+\xi_{0}\right), \\
& \beta\left(\alpha^{2}-\omega^{2}\right)>0, \\
& u_{3}(x, t)= \\
& \frac{\beta}{2 \gamma}\left[-1+3 \tanh ^{2}\left(\frac{1}{2} \sqrt{\frac{\beta}{\omega^{2}-\alpha^{2}}}(x-\omega t)+\xi_{0}\right)\right], \\
& \beta\left(\omega^{2}-\alpha^{2}\right)>0,
\end{aligned}
$$

where $\xi_{0}=\tanh ^{-1}\left(C_{1} / C_{2}\right)$, and the solution functions (29) and (31) become

$$
\begin{aligned}
& u_{2}(x, t)=\frac{3 \beta}{2 \gamma} \csc ^{2}\left(\frac{1}{2} \sqrt{\frac{-\beta}{\alpha^{2}-\omega^{2}}}(x-\omega t)+\xi_{0}\right), \\
& \beta\left(\alpha^{2}-\omega^{2}\right)<0, \\
& u_{4}(x, t)= \\
& \frac{\beta}{2 \gamma}\left[-1-3 \cot ^{2}\left(\frac{1}{2} \sqrt{\frac{-\beta}{\omega^{2}-\alpha^{2}}}(x-\omega t)+\xi_{0}\right)\right], \\
& \beta\left(\omega^{2}-\alpha^{2}\right)<0,
\end{aligned}
$$

where $\xi_{0}=\tan ^{-1}\left(C_{1} / C_{2}\right)$.

\section{Conclusion}

In the present paper, the auxiliary equation method, the so-called $\left(G^{\prime} / G\right)$-expansion method, has been tested by applying it successfully to two nonlinear equations of mathematical physics. The exact traveling wave solutions determined in this paper are more general and do not appear in [18-24] where the authors used various nonlinear analytical techniques. However, assigning values to arbitrary constants we have observed that it is possible to get some known analytical solutions. Our results are of general interest in physics and should be readily verified. Moreover, comparing with other methods in the literature, our method has the advantage that it provides both solitary and periodic traveling wave solutions. The free parameters, of course, might be related to initial conditions as well.

\section{Acknowledgement}

We would like to thank the anonymous referees for their useful and valuable suggestions. 
[1] M. L. Wang, Phys. Lett. A 213, 279 (1996).

[2] M. J. Ablowitz and H. Segur, Solitons and the Inverse Scattering Transform, SIAM Publications, Philadelphia 1981.

[3] R. Hirota, The Direct Method in Soliton Theory, Cambridge University Press, Cambridge 2004.

[4] R. M. Miura, Bäcklund Transformation, SpringerVerlag, New York 1973.

[5] Z. Yubin, W. Mingliang, and W. Yueming, Phys. Lett. A 308, 31 (2003).

[6] M. H. M. Moussa and R. M. El Shikh, Physica A 371, 325 (2006).

[7] A. M. Wazwaz, Appl. Math. Comput. 150, 365 (2004).

[8] W. Malfliet and W. Hereman, Phys. Scr. 54, 563 (1996).

[9] J. Weiss, M. Tabor, and G. Carnevale, J. Math. Phys. 24, 522 (1983).

[10] J. H. He, Chaos, Solitons and Fractals 26, 695 (2005).

[11] J. H. He and X. H. Wu, Chaos, Solitons and Fractals 30, 700 (2006).

[12] M. Wang, X. Li, and J. Zhang, Phys. Lett. A 372, 417 (2008).

[13] A. Bekir, Phys. Lett. A 372, 3400 (2008).
[14] J. Zhang, X. Wei, and Y. Lu, Phys. Lett. A 372, 3653 (2008).

[15] S. Zhang, J. L. Tong and W. Wang, Phys. Lett. A 372, 2254 (2008).

[16] L. Debnath, Nonlinear Partial Differential Equations for Scientists and Engineers, $2^{\text {nd }}$ ed., Birkhäuser, Boston 2005.

[17] E. Infeld and G. Rowlands, Nonlinear Waves, Solitons and Chaos, Cambridge University Press, Cambridge 2000.

[18] R. S. Banerjee, Int. J. Theoret. Phys. 32, 879 (1993).

[19] H. A. Abdusalam, Int. J. Comput. Methods 3, 371 (2006).

[20] H. Li and Y. Guo, Appl. Math. Comput. 180, 524 (2006).

[21] D. H. Kang, J. Central China Normal Univ. Natur. Sci. 31, 260 (1997).

[22] Sirendaoreji, Phys. Lett. A 363, 440 (2007).

[23] Sirendaoreji, Chaos, Solitons and Fractals 31, 943 (2007).

[24] A. M. Wazwaz, Chaos, Solitons and Fractals 28, 1005 (2006). 